\title{
Feeding Value of Copra Meal in Corn-Animal Protein-Based Diets and Enzyme Supplementation for Egg-Type Birds: Growth Performance Egg, Production and Fatty Acid Profile
}

\begin{tabular}{|c|c|}
\hline \multicolumn{2}{|c|}{$\begin{array}{l}{ }^{1} \text { School of Agriculture and Food Technology, University of the South Pacific, P. M. Bag Alafua, Apia WS1343, Samoa } \\
{ }^{2} \text { Vanuatu Agriculture College and Livestock-Based Integrated Farm, Luganville, P. O. Box 218, Santo, Vanuatu } \\
{ }^{*} \text { Corresponding author }\end{array}$} \\
\hline ARTICLE INFO & A B S T R A C T \\
\hline $\begin{array}{l}\text { Keywords: } \\
\text { Alternative ingredients } \\
\text { Diet composition } \\
\text { Enzyme supplementation } \\
\text { Egg quality } \\
\text { Layer performance }\end{array}$ & $\begin{array}{l}\text { A study investigated the effect of enzyme supplementation of copra meal in corn-animal protein- } \\
\text { based diets on pullet growth performance, egg production and fatty acid composition in laying hens. } \\
\text { A total of } 144,57 \text { day-old and } 20 \text { week-old Shaver Brown pullets were assigned to } 8 \text { diets, } 2 \text { controls } \\
\text { (no copra meal) with and without enzyme and } 6 \text { diets containing copra meal at } 150,300 \text { and } 450 \\
\mathrm{~g} / \mathrm{kg} \text { with and without enzyme. The experiment was laid as a factorial arrangement ( } 4 \text { copra meal } \times 2 \\
\text { enzyme) in completely randomised design with } 3 \text { replicates of } 6 \text { birds each per cage for pullet and } \\
\text { laying hens. Pullet results showed reduced feed intake on the control diet with enzyme compared to } \\
150,450 \mathrm{~g} / \mathrm{kg} \text { diets and } 300 \mathrm{~g} / \mathrm{kg} \text { diet without enzyme. In the main effects, weight gain was reduced } \\
\text { on } 300 \mathrm{~g} / \mathrm{kg} \text { copra meal diet. Enzyme supplementation had no effect on growth parameters of pullets. } \\
\text { Laying hens results showed significant interaction effects on feed intake and feed conversion ratio } \\
\text { but other performance parameters were unaffected by the interaction. Monounsaturated fatty acid of } \\
\text { the egg increased on all copra meal diets, saturated fatty acids increased on } 450 \mathrm{~g} / \mathrm{kg} \text { copra meal and } \\
\text { enzyme supplementation reduced this but interaction had no effect on poly-unsaturated fatty acids. } \\
\text { In the main effects, higher egg shape index and deeper yolk colour were observed on } 450 \mathrm{~g} / \mathrm{kg} \text { copra } \\
\text { meal diet. Inclusion of copra meal at } 450 \mathrm{~g} / \mathrm{kg} \text { increased saturated and monounsaturated fatty acid } \\
\text { of the egg but copra meal level had no effect on polyunsaturated fatty acid. Enzyme supplementation } \\
\text { had no effect on egg parameters reduced saturated fatty acid, increased monounsaturated fatty acid } \\
\text { without affecting polyunsaturated fatty acid. In corn-animal protein-based diets, copra meal can be } \\
\text { included in pullet and laying hens diets at } 450 \mathrm{~g} / \mathrm{kg} \text { without compromising pullet growth } \\
\text { performance, egg production and quality. }\end{array}$ \\
\hline
\end{tabular}

\author{
Q siakaciaria@uspocji
}


study investigated the effect of enzyme supplementation of corn-animal protein-based diets containing CM on the performance of laying hens. The following hypotheses were tested:

i. When fed with animal protein sources, copra meal level will not affect performance of laying hens or fatty acid composition of eggs.

ii. Supplementation of diets with enzyme will have no beneficial effects.

\section{Materials and Methods}

\section{Experimental Site}

The investigation consisted of two experiments and was carried out at Ratish Poultry farm, Baulevu road, Nausori, Fiji. The research committee of the University of the South Pacific approved the experimental protocol.

\section{Experimental Protein Sources}

The protein sources, fish meal (FM), meat and bone meal (MBM) were purchased locally from Fiji Meat Industries Board (Abattoir) and copra meal (CM) from Fiji Cooperative Dairy Company Limited and analysed for proximate composition, amino acid (AA) and fatty acid profile (Tables 1 and 2). Corn-animal protein-based diets were formulated for each of the experiment.

\section{Experimental Diets}

Eight pullet and layer mash diets based on FM and MBM as major protein sources were formulated to contain 15 and $17 \% \mathrm{CP}$ (crude protein) respectively for the experiment (Table 3 and 4). The diets consisted of 2 controls with no CM with and without enzyme and 6 other diets containing $\mathrm{CM}$ at 150,300 and $450 \mathrm{~g} / \mathrm{kg}$ with and without enzyme. The enzyme used in this study was Challenzyme 1309A from Beijing Challenge BioTechnology Company Limited with 8 activities (xylanase $15000 \mathrm{U} / g$, protease $8000 \mathrm{U} / g$, pectinase $500 \mathrm{U} / \mathrm{g}$, cellulase $300 \mathrm{U} / \mathrm{g}$, ß-glucanase $800 \mathrm{U} / \mathrm{g}$, , -mannanase $100 \mathrm{U} / \mathrm{g}, \alpha$-galactosidase $100 \mathrm{U} / \mathrm{g}$ and amylase $500 \mathrm{U} / \mathrm{g}$ ) (Challenzyme, 2016). Challenzyme was included at 300 g/tonne diet. All diets were fed as mash to the experimental birds.

\section{Experimental Birds and Management}

One hundred and sixty day-old Shaver Brown chicks were purchased from Pacific Feeds Limited, Suva, Fiji and warm brooded together on deep litter with wood shavings as litter material for the first 56 days, during which they were fed commercial chick starter feed. On day 57, 144 pullets were weighed and allotted to 24 rearing cages $(65.5 \mathrm{~cm} \times 50 \mathrm{~cm} \times 35.5 \mathrm{~cm})$ and fed 8 dietary treatments replicated 3 times with 6 birds per replicate in a completely randomized design. The experiment was laid out in a factorial arrangement of 4 levels of CM and 2 levels of enzyme for both pullet and laying hen experiments.

Pullets were vaccinated against Mareks, Infectious Bronchitis and Fowl Cholera at 8, 10 and 12 weeks respectively. On 15 th week, a total of 144 point of lay Shaver Brown pullets (140 day-old) were weighed nearest to mean weight $(1,206.9 \pm 34.5 \mathrm{~g})$ and transferred to layer cages and allocated to 72 cages with 2 birds per cage $(38.5 \mathrm{~cm} \times 35 \mathrm{~cm} \times 40 \mathrm{~cm})$. Feed and water were provided ad-libitum throughout the duration of the experiment to pullets and laying hens for 80 and 105 days respectively. The lighting programme for laying hens was $14 \mathrm{~h}$ as per (Shaver management guide, 2016). Stressol was added to drinking water for laying hens to reduce heat stress during the experiment.

\section{Data Collection}

\section{Growth Performance}

Feed intake (FI) was calculated by difference between the quantity fed and the leftover. Weight gain (WG) was obtained by difference between the initial and final weights. Feed conversion ratio (FCR) was derived as the ratio of feed consumed to weight gained in pullets.

\section{Egg Production and Quality Measurements}

Eggs produced were collected and counted per cage and hen-day production (HDP) calculated as:

$$
\mathrm{HDP}=(\text { eggs collected }) /(\text { hens present }) \times 100
$$

Sample eggs were weighed weekly per cage using a digital scale (Jadever JKH-500 series, Smartfox, Auckland, NZ) sensitive to $0.1 \mathrm{~g}$ and egg mass (EM) calculated as:

$$
\mathrm{EM}=\text { eggs collected } \times \text { mean egg weight }(\mathrm{g})
$$

Feed conversion ratio (FCR) was calculated as the ratio of unit feed consumed to unit egg produced as:

$$
\text { FCR }=\text { feed consumed/eggs mass }
$$

Sample eggs from each treatment were used for egg quality measurements on a weekly basis. Egg weight was taken using a digital scale sensitive to $0.1 \mathrm{~g}$. Egg length and width were measured using a digital Vernier caliper and shape index (SI) calculated as:

$$
\text { SI= Egg width/egg length } \times 100
$$

Eggs were then broken on a glass surface to separate the yolk carefully from the albumin. Yolk colour was determined using a Roche yolk colour fan. Albumin height was measured using a tripod spherometer. Shell thickness was taken as the mean of 3 measurements (broad end, middle and narrow end) using a digital Vernier caliper. Assessment of egg freshness was based on the Haugh unit (HU). Haugh unit (HU) was calculated according to Raymond Haugh (1937) cited in Eisen et al., 1962) as:

$$
\mathrm{HU}=100 \times \log \left(\mathrm{h}-1.7 \mathrm{w}^{0.37}+7.6\right)
$$

Where $\mathrm{h}=$ height of the albumen and $\mathrm{w}=$ weight of the egg. At the end of the experiment, a total of 32 eggs (4 eggs per treatment) were randomly selected for fatty acid composition at the Institute of Applied Sciences laboratory, University of the South Pacific, Laucala Campus, Suva. 


\section{Data Analysis}

Proximate analysis (dry matter, crude protein, crude fibre and fat was done according to (AOAC, 1990) procedures. Amino acid profile was determined using the Performic acid oxidation method (AOAC, 1997). Total fat was determined according to AOAC (2012) (ID 922.06) modified method and the fatty acid profiling done using
Hewlett Packard 6890® gas chromatograph (Sukhija and Palmquist, 1988).Total (TDF), soluble (SDF) and insoluble detergent fibre (IDF) was analysed using Megazyme as per AOAC 991.45.Neutral detergent fibre (NDF) and acid detergent fibre (ADF) were analysed using Tecator Fibertec according to AOAC 2002.04 and AOAC 973.18, respectively.

Table 1. Composition of the experimental protein sources in selected constituents

\begin{tabular}{l|ccc}
\hline \multirow{2}{*}{ Constituents $(\mathrm{g} / \mathrm{kg})$} & \multicolumn{3}{|c}{ Protein sources } \\
\cline { 2 - 4 } & FM & MBM & CM \\
\hline ME MJ/kg & 11.31 & 13.44 & 10.88 \\
Dry matter & 725 & 879 & 887 \\
Crude protein & 531 & 481 & 184 \\
Ether extract & 106 & 253 & 120 \\
Ash & 117 & 144 & 49 \\
IDF & - & - & 402 \\
SDF & - & - & 17 \\
TDF & - & - & 419 \\
Crude fibre & 5 & 21 & 189 \\
NDF & 222 & 250 & 441 \\
ADF & 26 & 82 & 271 \\
\hline
\end{tabular}

ME: metabolisable energy; FM: fish meal; MBM: meat and bone meal; CM: copra meal, IDF: insoluble dietary fibre; SDF: soluble dietary fibre TDF: total dietary fibre; NDF: neutral detergent fibre; ADF: acid detergent fibre

Table 2. Fatty acid composition and amino acid profile of the experimental protein sources

\begin{tabular}{|c|c|c|c|c|c|}
\hline Fatty acids (g/100g DM) & Systematic name & Formulae & FM & MBM & $\mathrm{CM}$ \\
\hline $\mathrm{C}_{6: 0}$ Caproic & Hexanoic acid & $\mathrm{C}_{6} \mathrm{H}_{12} \mathrm{O}_{2}$ & ND & 0.01 & 0.12 \\
\hline $\mathrm{C}_{8: 0}$ Caprytic & Octanoic acid & $\mathrm{C}_{8} \mathrm{H}_{16} \mathrm{O}_{2}$ & ND & 0.01 & 1.26 \\
\hline $\mathrm{C}_{10: 0}$ Capric & Decanoic acid & $\mathrm{C}_{10} \mathrm{H}_{20} \mathrm{O}_{2}$ & $<0.01$ & 0.02 & 0.89 \\
\hline $\mathrm{C}_{11: 0}$ Undecylic & Undecanoic acid & $\mathrm{C}_{11} \mathrm{H}_{22} \mathrm{O}_{2}$ & $<0.01$ & $<0.01$ & $<0.01$ \\
\hline $\mathrm{C}_{12: 0}$ Lauric & Dodecanoic acid & $\mathrm{C}_{12} \mathrm{H}_{24} \mathrm{O}_{2}$ & $<0.01$ & 0.02 & 6.41 \\
\hline $\mathrm{C}_{13: 0}$ Tridecylic & Trideanoic acid & $\mathrm{C}_{13} \mathrm{H}_{26} \mathrm{O}_{2}$ & $<0.01$ & 0.01 & 0.01 \\
\hline $\mathrm{C}_{14: 0}$ Myristic & Tetradecanoic and & $\mathrm{C}_{14} \mathrm{H}_{28} \mathrm{O}_{2}$ & 0.21 & 0.69 & 2.43 \\
\hline $\mathrm{C}_{16: 0}$ Palmitic & Hexadecanoic acid & $\mathrm{C}_{16} \mathrm{H}_{32} \mathrm{O}_{2}$ & 1.93 & 5.99 & 1.21 \\
\hline $\mathrm{C}_{17: 0}$ Margaric & Heptadecanoic acid & $\mathrm{C}_{17} \mathrm{H}_{34} \mathrm{O}_{2}$ & 0.13 & 0.43 & 0.01 \\
\hline $\mathrm{C}_{18: 0-3}$ Stearic & Octadecanoic acid & $\mathrm{C}_{18} \mathrm{H}_{36} \mathrm{O}_{2}$ & 2.07 & 12.66 & 1.55 \\
\hline $\mathrm{C}_{20: 1-5}$ Arachidic & Eicosanoic acid & $\mathrm{C}_{20} \mathrm{H}_{40} \mathrm{O}_{2}$ & 0.77 & 0.52 & 0.01 \\
\hline $\mathrm{C}_{21: 0}$ Heneicosylic & Heneicosanoic acid & $\mathrm{C}_{21} \mathrm{H}_{42} \mathrm{O}_{2}$ & 0.02 & 0.04 & 0.02 \\
\hline $\mathrm{C}_{22: 0-6}$ Behenic & Docosanoic acid & $\mathrm{C}_{22} \mathrm{H}_{44} \mathrm{O}_{2}$ & 2.3 & 1.19 & 0.01 \\
\hline $\mathrm{C}_{23: 0}$ Tricosylic & Tricosanoic acid & $\mathrm{C}_{23} \mathrm{H}_{46} \mathrm{O}_{2}$ & 0.01 & 0.01 & ND \\
\hline $\mathrm{C}_{24: 0-1}$ Lignoceric & Tetracosanoic acid & $\mathrm{C}_{24} \mathrm{H}_{48} \mathrm{O}_{2}$ & 0.1 & 0.07 & 0.02 \\
\hline \multicolumn{6}{|l|}{ Amino acids (mg/100mg DM) } \\
\hline Aspartic acid & L-Aspartic acid & $\mathrm{C}_{4} \mathrm{H}_{7} \mathrm{NO}_{4}$ & 4.58 & 3.61 & 1.48 \\
\hline Threonine & L-Threonine & $\mathrm{C}_{4} \mathrm{H}_{9} \mathrm{NO}_{3}$ & 2.16 & 1.67 & 0.56 \\
\hline Serine & L-Serine & $\mathrm{C}_{3} \mathrm{H}_{7} \mathrm{NO}_{3}$ & 2.13 & 1.90 & 0.79 \\
\hline Glutamic acid & L-glutamic acid & $\mathrm{C}_{5} \mathrm{H}_{9} \mathrm{NO}_{4}$ & 6.68 & 5.63 & 3.12 \\
\hline Proline & L-Proline & $\mathrm{C}_{5} \mathrm{H}_{9} \mathrm{NO}_{2}$ & 2.84 & 3.20 & 0.62 \\
\hline Glycine & 2-Aminoethanoic acid & $\mathrm{C}_{2} \mathrm{H}_{5} \mathrm{NO}_{2}$ & 4.61 & 5.21 & 0.81 \\
\hline Alanine & L-Alanine & $\mathrm{C}_{3} \mathrm{H}_{7} \mathrm{NO}_{2}$ & 3.44 & 3.16 & 0.80 \\
\hline Valine & L-Valine & $\mathrm{C}_{5} \mathrm{H}_{11} \mathrm{NO}_{2}$ & 2.27 & 1.95 & 0.89 \\
\hline Isoleucine & L-Isoleucine & $\mathrm{C}_{6} \mathrm{H}_{13} \mathrm{NO}_{2}$ & 1.85 & 1.45 & 0.56 \\
\hline Leucine & L-leucine & $\mathrm{C}_{6} \mathrm{H}_{13} \mathrm{NO}_{2}$ & 3.46 & 2.87 & 1.10 \\
\hline Tyrosine & L-tyrosine & $\mathrm{C}_{9} \mathrm{H}_{11} \mathrm{NO}_{3}$ & 1.50 & 1.18 & 0.47 \\
\hline Phenylalanine & L-Phenylalanine & $\mathrm{C}_{9} \mathrm{H}_{11} \mathrm{NO}_{2}$ & 1.87 & 1.56 & 0.74 \\
\hline Histidine & L-Histidine & $\mathrm{C}_{6} \mathrm{H}_{9} \mathrm{~N}_{3} \mathrm{O}_{2}$ & 1.50 & 1.26 & 0.39 \\
\hline Lysine & L-Lysine & $\mathrm{C}_{6} \mathrm{H}_{14} \mathrm{~N}_{2} \mathrm{O}_{2}$ & 3.84 & 2.75 & 0.64 \\
\hline Arginine & L-arginine & $\mathrm{C}_{6} \mathrm{H}_{14} \mathrm{~N}_{4} \mathrm{O}_{2}$ & 3.29 & 3.12 & 2.35 \\
\hline Cysteine & L-Cysteine & $\mathrm{C}_{3} \mathrm{H}_{7} \mathrm{NO}_{2} \mathrm{~S}$ & 0.36 & 0.25 & 0.28 \\
\hline Methionine & DL-methionine & $\mathrm{C}_{5} \mathrm{H}_{11} \mathrm{NO}_{2} \mathrm{~S}$ & 1.41 & 0.90 & 0.31 \\
\hline Tryptophan & L-Tryptophan & $\mathrm{C}_{11} \mathrm{H}_{12} \mathrm{~N}_{2} \mathrm{O}_{2}$ & 0.46 & 0.30 & 0.15 \\
\hline
\end{tabular}

FM: fish meal; MBM: meat and bone meal; CM: copra meal; DM: dry matter; ND: not detected 
Table 3. Ingredient composition and calculated analysis of pullet grower diets

\begin{tabular}{|c|c|c|c|c|c|c|c|c|}
\hline \multirow{2}{*}{ Ingredients(g/kg) } & \multicolumn{4}{|c|}{ CM $(\mathrm{g} / \mathrm{kg})$ with no enzyme } & \multicolumn{4}{|c|}{ CM $(\mathrm{g} / \mathrm{kg})$ with enzyme } \\
\hline & 0 & 150 & 300 & 450 & 0 & 150 & 300 & 450 \\
\hline Corn & 543.9 & 463.5 & 382.9 & 302.3 & 543.7 & 463.3 & 382.7 & 302.1 \\
\hline Wheat middling & 272 & 231.7 & 191.5 & 151.2 & 271.9 & 231.6 & 191.3 & 151 \\
\hline Tuna fish meal & 42.4 & 32.5 & 22.6 & 12.7 & 42.4 & 32.5 & 22.6 & 12.7 \\
\hline Meat \& bone meal & 84.9 & 65.1 & 45.2 & 25.4 & 84.9 & 65.1 & 45.3 & 25.5 \\
\hline Copra meal & 0 & 150 & 300 & 450 & 0 & 150 & 300 & 450 \\
\hline Sand & 30 & 30 & 30 & 30 & 30 & 30 & 30 & 30 \\
\hline Limestone & 20 & 20 & 20 & 20 & 20 & 20 & 20 & 20 \\
\hline *Premix & 2.5 & 2.5 & 2.5 & 2.5 & 2.5 & 2.5 & 2.5 & 2.5 \\
\hline Lysine & 0.8 & 1 & 1.5 & 2 & 0.8 & 1 & 1.5 & 2 \\
\hline Methionine & 0.5 & 0.7 & 0.8 & 0.9 & 0.5 & 0.7 & 0.8 & 0.9 \\
\hline Enzyme & 0 & 0 & 0 & 0 & 0.3 & 0.3 & 0.3 & 0.3 \\
\hline Salt & 3 & 3 & 3 & 3 & 3 & 3 & 3 & 3 \\
\hline \multicolumn{9}{|l|}{ Calculated analysis } \\
\hline Crude protein $(\%)$ & 15 & 15 & 15 & 15 & 15 & 15 & 15 & 15 \\
\hline $\mathrm{ME}(\mathrm{MJ} / \mathrm{kg})$ & 11.9 & 11.8 & 11.7 & 11.6 & 11.9 & 11.8 & 11.7 & 11.6 \\
\hline Lysine $(\mathrm{g} / \mathrm{kg})$ & 7.9 & 7.7 & 7.6 & 7.6 & 7.9 & 7.7 & 7.6 & 7.6 \\
\hline Methionine $(\mathrm{g} / \mathrm{kg})$ & 3.5 & 3.6 & 3.7 & 3.8 & 3.5 & 3.6 & 3.7 & 3.8 \\
\hline
\end{tabular}

*Premix (Vitamin and mineral) Bio-mix supplied/kg diet, vitamin A: $10000 \mathrm{IU}$, vitamin D3: $2000 \mathrm{IU}$, vitamin E: $23 \mathrm{mg}$, niacin: $27.5 \mathrm{mg}$, vitamin B1: $1.8 \mathrm{mg}$, B2: 5mg, B6: 3mg, B12: 0.015mg, vitamin K: 3.2mg, pantothenic acid:7.7mg, biotin:0.06mg, folic acid: $0.75 \mathrm{mg}$, choline chloride: 300mg, cobalt: $0.2 \mathrm{mg}$, copper: $3 \mathrm{mg}$.iodine: $1 \mathrm{mg}$, iron: $20 \mathrm{mg}$, manganese: $40 \mathrm{mg}$, selenium: $0.2 \mathrm{mg}$, zinc: $30 \mathrm{mg}$, anti-oxidant: $1.25 \mathrm{mg}$; CM: Copra meal; ME: Metabolisable Energy

Table 4. Ingredient composition and calculated analysis of the layer diets

\begin{tabular}{|c|c|c|c|c|c|c|c|c|}
\hline \multirow{2}{*}{ Ingredients $(\mathrm{g} / \mathrm{kg})$} & \multicolumn{4}{|c|}{$\mathrm{CM}(\mathrm{g} / \mathrm{kg})$ with no enzyme } & \multicolumn{4}{|c|}{ CM $(\mathrm{g} / \mathrm{kg})$ with enzyme } \\
\hline & 0 & 150 & 300 & 450 & 0 & 150 & 300 & 450 \\
\hline Corn & 499.2 & 421 & 342.1 & 263.4 & 499.1 & 420.5 & 341.9 & 263.2 \\
\hline Wheat middling & 249.6 & 210.1 & 171 & 131.7 & 249.4 & 210.3 & 170.9 & 131.6 \\
\hline Tuna fish meal & 51.5 & 40.6 & 29.7 & 18.8 & 51.5 & 40.6 & 29.7 & 18.8 \\
\hline Meat \& bone meal & 102.9 & 81.1 & 59.4 & 37.7 & 102.9 & 81.1 & 59.4 & 37.7 \\
\hline Copra meal & 0 & 150 & 300 & 450 & 0 & 150 & 300 & 450 \\
\hline Sand & 40 & 40 & 40 & 40 & 40 & 40 & 40 & 40 \\
\hline Limestone & 50 & 50 & 50 & 50 & 50 & 50 & 50 & 50 \\
\hline *Premix & 2.5 & 2.5 & 2.5 & 2.5 & 2.5 & 2.5 & 2.5 & 2.5 \\
\hline Lysine & 0.8 & 1 & 1.5 & 2 & 0.8 & 1 & 1.5 & 2 \\
\hline Methionine & 0.5 & 0.7 & 0.8 & 0.9 & 0.5 & 0.7 & 0.8 & 0.9 \\
\hline Enzyme & 0 & 0 & 0 & 0 & 0.3 & 0.3 & 0.3 & 0.3 \\
\hline Salt & 3 & 3 & 3 & 3 & 3 & 3 & 3 & 3 \\
\hline \multicolumn{9}{|l|}{ Calculated analysis } \\
\hline Crude protein $(\%)$ & 17 & 17 & 17 & 17 & 17 & 17 & 17 & 17 \\
\hline $\mathrm{ME}(\mathrm{MJ} / \mathrm{kg})$ & 12.2 & 12 & 11.8 & 11.5 & 12.2 & 12 & 11.7 & 11.5 \\
\hline Lysine $(\mathrm{g} / \mathrm{kg})$ & 8.7 & 8.2 & 8.1 & 8.0 & 8.7 & 8.2 & 8.1 & 8.0 \\
\hline Methionine $(\mathrm{g} / \mathrm{kg})$ & 3.6 & 3.8 & 3.8 & 3.9 & 3.6 & 3.8 & 3.8 & 3.9 \\
\hline Calcium $(\mathrm{g} / \mathrm{kg})$ & 32.8 & 30.1 & 27.4 & 24.7 & 32.8 & 30.1 & 27.4 & 24.7 \\
\hline Phosphorus (g/kg) & 10.7 & 9.5 & 8.3 & 7.1 & 10.7 & 9.5 & 8.3 & 7.1 \\
\hline $\mathrm{Ca}: \mathrm{P}$ & $3.1: 1$ & $3.2: 1$ & $3.3: 1$ & $3.5: 1$ & $3.1: 1$ & $3.2: 1$ & $3.3: 1$ & $3.5: 1$ \\
\hline
\end{tabular}

*Premix (Vitamin and mineral) Bio-mix supplied/kg diet, vitamin A: $10000 \mathrm{IU}$, vitamin D3: $2000 \mathrm{IU}$, vitamin E: 23mg, niacin: $27.5 \mathrm{mg}$, vitamin B1: $1.8 \mathrm{mg}$, B2: 5mg, B6: 3mg, B12: 0.015mg, vitamin K: 3.2mg, pantothenic acid:7.7mg, biotin:0.06mg, folic acid: $0.75 \mathrm{mg}$, choline chloride: 300mg, cobalt: $0.2 \mathrm{mg}$, copper: $3 \mathrm{mg}$.iodine: $1 \mathrm{mg}$, iron: $20 \mathrm{mg}$, manganese: $40 \mathrm{mg}$, selenium: $0.2 \mathrm{mg}$, zinc: $30 \mathrm{mg}$, anti-oxidant: $1.25 \mathrm{mg}$; CM: Copra meal; ME: Metabolisable energy.

Gross energy (solid) was determined in the Bomb calorimeter and Metabolisable energy (ME) calculated according to Fisher and Boorman (1986) as:

$$
\mathrm{ME}(\mathrm{Kcal} / \mathrm{Kg})=37 \times \mathrm{CP}+81 \times \mathrm{EE}+35.5 \times \mathrm{NFE} \text {. }
$$

Where $\mathrm{CP}=$ crude protein; $\mathrm{NFE}=$ nitrogen-free extract and $\mathrm{EE}=$ ether extract.

\section{Statistical Analysis}

Data collected were subjected to ANOVA (Steel and Torrie, 1980) of a factorial arrangement using the GLM of SPSS (Windows, version 22.0; IBM Corp, Armonk, NY, USA) (SPSS, 2013). Individual were the experimental units for weight change whereas cages were the experimental units for feed intake.

Treatment means were compared using the Least Significant Difference (LSD) and differences considered significant when $(\mathrm{P}<0.05)$. 


\section{Results}

\section{Pullet Growth Performances}

From the growth performance results of pullets (Table 5) WG and FCR were not affected by the interaction of $\mathrm{CM}$ and enzyme but there was a significant interaction effect on FI $(\mathrm{P}<0.05)$. Enzyme supplementation reduced the intake of the control diet compared to 150 and 450 $\mathrm{g} / \mathrm{kg}$ and $300 \mathrm{~g} / \mathrm{kg} \mathrm{CM}$ without enzyme $(\mathrm{P}<0.05)$.

There was no significant interaction effect on FI between the negative control and CM based diets $(\mathrm{P}>0.05)$. In the main effect, weight gain was depressed on $300 \mathrm{~g} / \mathrm{kg} \mathrm{CM} \operatorname{diet}(\mathrm{P}<0.05)$. A better FCR was observed on control compared to the $\mathrm{CM}$ diets $(\mathrm{P}<0.05)$. Enzyme addition did not affect any of the performance parameters studied $(\mathrm{P}>0.05)$. No mortality was recorded throughout the pullet experiment.

\section{Laying Performance}

The egg performance results are presented in Table 6. No mortality was recorded throughout layer experiment. Feed intake and FCR showed significant interaction effects $(\mathrm{P}<0.05)$ but egg mass remained unaffected $(\mathrm{P}>0.05)$. Feed intake significantly reduced on the $300 \mathrm{~g}$ $\mathrm{CM} / \mathrm{kg}$ with enzyme compared to the other CM-based diets $(\mathrm{P}<0.05)$. Feed conversion ratio was poorer on 300 g CM without enzyme and 450g CM with enzyme $(\mathrm{P}<0.05)$ compared to control diets and 150g CM without enzyme.
In the main effects, CM level had no effects on FI, HDP, MEW, EM, HU and shell thickness (P>0.05). Feed conversion ratio increased above $150 \mathrm{~g} \mathrm{CM} / \mathrm{kg}(\mathrm{P}<0.05)$. Lower shape index was observed on $300 \mathrm{~g} \mathrm{CM} / \mathrm{kg}$ compared to $450 \mathrm{~g} \mathrm{CM} / \mathrm{kg}(\mathrm{P}<0.05)$. A deeper yolk colour was recorded on the $450 \mathrm{~g} \mathrm{CM} / \mathrm{kg}(\mathrm{P}<0.05)$ but yolk colour did not differ among the control, 150 and $300 \mathrm{~g} \mathrm{CM} / \mathrm{kg}$ $(\mathrm{P}>0.05)$. Enzyme supplementation had no effects on any of the performance traits evaluated $(\mathrm{P}>0.05)$.

\section{Fatty Acid Composition of Eggs}

The fatty acids SFA (saturated), MUFA (monounsaturated) and PUFA (polyunsaturated) compositions $(\mathrm{g} / 100 \mathrm{~g})$ of the eggs are presented in Table 7. Saturated fatty acid and MUFA composition was significantly affected by the interaction $(\mathrm{P}<0.05)$ but PUFA was unaffected (P>0.05). Lower values of SFA were recorded on the control and the $300 \mathrm{~g} \mathrm{CM}$ with and without enzyme and higher MUFA on the $450 \mathrm{~g} \mathrm{CM}$ with enzyme $(\mathrm{P}<0.05)$. In the main effects, higher values of SFA and MUFA were observed on $450 \mathrm{~g} / \mathrm{kg} \mathrm{CM}(\mathrm{P}<0.05)$ but PUFA was not affected CM level (P>0.05). Enzyme supplementation reduced SFA and increased MUFA $(\mathrm{P}<0.05)$ but had no effect on PUFA composition $(\mathrm{P}>0.05)$.

Table 5. Growth performances of pullets fed increasing CM levels in corn-animal protein-based diets and Challenzyme supplementation

\begin{tabular}{|c|c|c|c|c|}
\hline \multicolumn{2}{|c|}{ Treatment } & \multirow{2}{*}{$\begin{array}{c}\text { FI } \\
\text { (kg/cage) }\end{array}$} & \multirow{2}{*}{$\begin{array}{c}\mathrm{WG} \\
\text { (kg/cage) }\end{array}$} & \multirow{2}{*}{ FCR } \\
\hline Copra & Enzyme & & & \\
\hline \multirow{2}{*}{0} & No & $24.44^{\mathrm{ab}}$ & 3.84 & 6.45 \\
\hline & Yes & $23.16^{\mathrm{b}}$ & 4.44 & 5.22 \\
\hline \multirow{2}{*}{150} & No & $25.42^{\mathrm{a}}$ & 4.21 & 6.07 \\
\hline & Yes & $26.16^{\mathrm{a}}$ & 3.77 & 6.95 \\
\hline \multirow{2}{*}{300} & No & $26.45^{\mathrm{a}}$ & 3.45 & 7.68 \\
\hline & Yes & $24.23^{\mathrm{ab}}$ & 3.71 & 6.68 \\
\hline \multirow{2}{*}{450} & No & $25.28^{\mathrm{a}}$ & 4.05 & 6.29 \\
\hline & Yes & $27.11^{\mathrm{a}}$ & 4.29 & 6.51 \\
\hline SEM & & 0.691 & 0.269 & 0.534 \\
\hline \multicolumn{5}{|c|}{ Main effects } \\
\hline \multicolumn{5}{|l|}{ Copra } \\
\hline 0 & & 23.80 & $4.14^{\mathrm{a}}$ & $5.84^{b}$ \\
\hline 150 & & 25.79 & $3.99^{\mathrm{a}}$ & $6.51^{\mathrm{a}}$ \\
\hline 300 & & 25.34 & $3.58^{\mathrm{b}}$ & $7.18^{\mathrm{a}}$ \\
\hline 450 & & 26.20 & $4.17^{\mathrm{a}}$ & $6.40^{\mathrm{a}}$ \\
\hline \multicolumn{5}{|l|}{ Enzyme } \\
\hline No & & 25.40 & 3.89 & 6.62 \\
\hline Yes & & 25.17 & 4.05 & 6.34 \\
\hline \multicolumn{5}{|l|}{ Probabilities } \\
\hline Enzyme & & 0.057 & 0.048 & 0.035 \\
\hline Copra & & 0.644 & 0.401 & 0.466 \\
\hline Copra*Enzyme & & 0.038 & 0.306 & 0.195 \\
\hline
\end{tabular}

FCR: feed conversion ratio; FI: feed intake; WG: weight gain; SEM: standard error of mean; a, b, c: values within the column with different superscripts differ significantly $(\mathrm{P}<0.05)$. 
Table 6. Performance of laying hens fed varying CM levels in corn-animal protein diets and Challenzyme supplementation

\begin{tabular}{|c|c|c|c|c|c|c|c|c|c|c|}
\hline \multicolumn{2}{|c|}{ Treatment } & \multirow{2}{*}{$\begin{array}{c}\text { FI } \\
(\mathrm{kg})\end{array}$} & \multirow{2}{*}{$\begin{array}{l}\text { HDP } \\
(\%)\end{array}$} & \multirow{2}{*}{$\begin{array}{c}\text { MEW } \\
(\mathrm{g})\end{array}$} & \multirow{2}{*}{$\begin{array}{l}\text { EM } \\
(\mathrm{kg})\end{array}$} & \multirow{2}{*}{$\begin{array}{c}\text { FCR } \\
\text { (FI:EM) }\end{array}$} & \multirow{2}{*}{$\mathrm{HU}$} & \multirow{2}{*}{ SI } & \multirow{2}{*}{$\mathrm{YC}$} & \multirow{2}{*}{$\begin{array}{c}\mathrm{ST} \\
(\mathrm{mm})\end{array}$} \\
\hline Copra & Enzyme & & & & & & & & & \\
\hline \multirow{2}{*}{0} & No & $35.07^{\mathrm{ab}}$ & 73.9 & 48.3 & 12.97 & $2.73^{\mathrm{c}}$ & 80.6 & 77.4 & 5.8 & 43 \\
\hline & Yes & $36.26^{\mathrm{a}}$ & 75.2 & 46.5 & 13.10 & $2.78^{c}$ & 75.8 & 78.0 & 5.8 & 46 \\
\hline \multirow{2}{*}{150} & No & $36.19^{a}$ & 81.9 & 47.2 & 13.61 & $2.66^{\mathrm{c}}$ & 79.9 & 78.3 & 5.0 & 41 \\
\hline & Yes & $38.43^{\mathrm{a}}$ & 72.6 & 47.7 & 12.00 & $3.24^{\mathrm{b}}$ & 81.0 & 77.2 & 5.0 & 41 \\
\hline \multirow{2}{*}{300} & No & $38.68^{\mathrm{a}}$ & 68.2 & 47.1 & 11.37 & $3.42^{\mathrm{a}}$ & 83.4 & 75.9 & 5.8 & 44 \\
\hline & Yes & $34.92^{\mathrm{b}}$ & 72.7 & 48.5 & 12.17 & $2.88^{\mathrm{bc}}$ & 82.1 & 76.5 & 5.0 & 41 \\
\hline \multirow{2}{*}{450} & No & $37.26^{\mathrm{a}}$ & 74.7 & 50.2 & 13.44 & $2.80^{\mathrm{bc}}$ & 80.2 & 78.9 & 7.5 & 44 \\
\hline & Yes & $37.49^{\mathrm{a}}$ & 65.6 & 47.4 & 11.07 & $3.44^{\mathrm{a}}$ & 86.5 & 78.8 & 5.8 & 42 \\
\hline \multicolumn{2}{|l|}{ SEM } & 0.923 & 3.721 & 1.347 & 0.828 & 0.169 & 2.687 & 0.702 & 0.589 & 0.018 \\
\hline \multicolumn{11}{|c|}{ Main effects } \\
\hline \multicolumn{11}{|l|}{ Copra } \\
\hline 0 & \multicolumn{2}{|c|}{35.66} & 74.5 & 47.4 & 13.04 & $2.75^{b}$ & 78.2 & $77.7^{\mathrm{ab}}$ & $5.8^{\mathrm{b}}$ & 44 \\
\hline 150 & \multicolumn{2}{|c|}{37.31} & 77.3 & 47.5 & 12.81 & $2.95^{\mathrm{ab}}$ & 80.5 & $77.8^{\mathrm{ab}}$ & $5.0^{\mathrm{b}}$ & 41 \\
\hline 300 & \multicolumn{2}{|c|}{36.80} & 70.5 & 47.8 & 11.77 & $3.15^{\mathrm{a}}$ & 82.8 & $76.2^{\mathrm{b}}$ & $5.4^{\mathrm{b}}$ & 43 \\
\hline 450 & \multicolumn{2}{|c|}{37.38} & 70.2 & 48.8 & 12.26 & $3.12^{\mathrm{a}}$ & 83.4 & $78.8^{\mathrm{a}}$ & $6.7^{\mathrm{a}}$ & 43 \\
\hline \multicolumn{11}{|l|}{ Enzyme } \\
\hline No & \multirow{2}{*}{\multicolumn{2}{|c|}{$\begin{array}{l}36.80 \\
36.77\end{array}$}} & 74.7 & 48.2 & 12.85 & 2.90 & 81.0 & 77.6 & 6.0 & 43 \\
\hline Yes & & & 71.6 & 47.5 & 12.09 & 3.08 & 81.4 & 77.6 & 5.4 & 43 \\
\hline \multicolumn{11}{|l|}{ Probabilities } \\
\hline Copra & & 0.209 & 0.706 & 0.441 & 0.013 & 0.242 & 0.015 & 0.046 & 0.407 \\
\hline Enzyme & \multirow{2}{*}{\multicolumn{2}{|c|}{$\begin{array}{l}0.967 \\
0.026\end{array}$}} & 0.250 & 0.480 & 0.210 & 0.149 & 0.864 & 0.979 & 0.153 & 0.797 \\
\hline Copra*Enzyme & & & 0.182 & 0.402 & 0.231 & 0.010 & 0.254 & 0.578 & 0.455 & 0.395 \\
\hline
\end{tabular}

FI: feed intake; EM: egg mass; HDP: hen day production; MEW: mean egg weight; FCR: feed conversion ratio; HU: Haugh Unit; SI: Shape index; YC: Yolk colour; ST: Shell Thickness SEM: standard error of mean; a, b, c: values within the column with different superscripts differ significantly $(\mathrm{P}<0.05)$

\section{Discussion}

\section{Chemical Analysis}

The CP content of the experimental CM $(184 \mathrm{~g} / \mathrm{kg})$ is comparable to $180-210 \mathrm{~g} / \mathrm{kg}$ reported by (Sundu et al., 2014; Diarra et al., 2018). Other authors (Devi and Diarra, 2017; Sundu et al., 2009; Sundu et al., 2006; Diarra et al., 2014) reported up to $250 \mathrm{~g} / \mathrm{kg} \mathrm{CP}$ in CM. The AA composition showed slightly higher profiles of lysine $(6.4 \mathrm{~g} / \mathrm{kg})$ and methionine $(3.1 \mathrm{~g} / \mathrm{kg})$ than the values (4.7 and $2.9 \mathrm{~g} / \mathrm{kg}$, respectively) reported by (Devi and Diarra, 2017). The experimental CM contained about $420 \mathrm{~g} / \mathrm{kg}$ total NSP. (Knudsen, 1997) also reported same value $(420 \mathrm{~g} / \mathrm{kg})$ of NSP in CM. However, other authors Devi and Diarra, 2017; Devi et al., 2019) reported higher NSP $(520-610 \mathrm{~g} / \mathrm{kg})$ in CM. Several factors including coconut variety, harvest age, drying process, oil extraction method and storage conditions affect the composition of CM (Sundu et al., 2006; Diarra et al., 2014; Panigrahi, 1992). The fatty acid composition of the experimental CM characterised by higher SFA and lower unsaturated fatty acids is in agreement with earlier reports (NRC, 1994; Boateng et al., 2016).

\section{Pullet Growth Performance}

Feed intake was reduced on the control diet with enzyme compared to 150 and $450 \mathrm{~g} / \mathrm{kg}$ diets and $300 \mathrm{~g} / \mathrm{kg}$ $\mathrm{CM}$ without enzyme. This may be attributed to increased nutrient availability resulting from enhanced hydrolysis of the low fibre control diet suggesting that the enzyme concentration was not sufficient enough to hydrolyse CM at these inclusion levels. Diarra et al. (2018) also reported higher FI in 56-132 days' old growing pullets fed $200 \mathrm{~g}$ $\mathrm{CM} / \mathrm{kg}$ with enzyme compared to control and attributed this to faster digesta transit in the gastro intestinal tract (GIT).The higher intake of the CM diets may be attributed to several factors including i) an attempt by the birds to meet their nutrient need, and ii) the beneficial effects of fibre on GIT development (Mateos et al., 2012) suggested that before the onset of egg production, dietary fibre improves GIT development in birds. Bouvarel and Nys (2013) and Pottguetter (2015) also confirmed that dietary $\mathrm{CF}$ is needed for stimulation of GIT development and FI at starter phase. In the main effect, the reduced WG on $300 \mathrm{~g}$ $\mathrm{CM} / \mathrm{kg}$ diet compared to other CM diets despite similar FI was not understood. The results of this study are in agreement with those of Diarra et al. (2018) who found reduced WG in pullets fed $200 \mathrm{~g} / \mathrm{kg} \mathrm{CM}$. Moorthy and Viswanathan; 2006; Moorthy and Viswanathan; 2010) also reported reduced $\mathrm{WG}$ and egg production of laying hens above $150-200 \mathrm{~g} / \mathrm{kg} \mathrm{CM}$ in the diet.Contrary to the findings of this study (Sundu et al., 2005) found improved WG in 6 weeks old Ross male broilers fed $300 \mathrm{~g} / \mathrm{kg} \mathrm{CM}$ with enzyme (Hemicel, mannanase $\AA$, $\beta$-mannanase and Allzyme SSF ${ }^{\text {) }}$ compared to diets without enzyme. The class of poultry, diet composition, enzyme source and concentration are all possible reasons for the differences in the utilisation of CM by poultry.

The improved WG on $450 \mathrm{~g} / \mathrm{kg}$ compared to $300 \mathrm{~g} / \mathrm{kg}$ $\mathrm{CM}$ diet in this study was not clear but possible enhanced gut health on this fibrous diet may be speculated. The poor FCR on CM diets compared to control diets was mainly attributed to the linear increase in FI on CM based diets compared to the control (Diarra et al.,2014; Diarra et al. (2015) also reported poor FCR in pullets fed CM diet at $200 \mathrm{~g} / \mathrm{kg}$ without enzyme. 
Table 7. Fatty acid compositions SFA, MUFA, PUFA (g/100g) of eggs of laying hens fed increasing CM levels in cornanimal protein-based diets with and without Challenzyme

\begin{tabular}{|c|c|c|c|c|}
\hline \multicolumn{2}{|c|}{ Treatment } & \multirow{2}{*}{ SFA } & \multirow{2}{*}{ MUFA } & \multirow{2}{*}{ PUFA } \\
\hline Copra & Enzyme & & & \\
\hline \multirow{2}{*}{0} & No & $2.77^{\mathrm{c}}$ & $1.07^{\mathrm{b}}$ & 0.095 \\
\hline & Yes & $3.60^{\mathrm{b}}$ & $0.80^{\mathrm{b}}$ & 0.096 \\
\hline \multirow{2}{*}{150} & No & $3.70^{\mathrm{b}}$ & $0.87^{\mathrm{b}}$ & 0.091 \\
\hline & Yes & $3.63^{\mathrm{b}}$ & $0.83^{\mathrm{b}}$ & 0.093 \\
\hline \multirow{2}{*}{300} & No & $2.57^{\mathrm{c}}$ & $0.80^{\mathrm{b}}$ & 0.092 \\
\hline & Yes & $2.67^{\mathrm{c}}$ & $0.73^{b}$ & 0.091 \\
\hline \multirow{2}{*}{450} & No & $4.80^{\mathrm{a}}$ & $0.97^{\mathrm{b}}$ & 0.095 \\
\hline & Yes & $3.87^{\mathrm{b}}$ & $2.17^{\mathrm{a}}$ & 0.097 \\
\hline & 0.152 & 0.117 & 0.009 \\
\hline \multicolumn{2}{|l|}{ SEM } & Main ef & & \\
\hline \multicolumn{5}{|l|}{ Copra } \\
\hline 0 & & $3.18^{c}$ & $0.93^{\mathrm{b}}$ & 0.094 \\
\hline 150 & & $3.67^{b}$ & $0.85^{b}$ & 0.092 \\
\hline 300 & & $2.62^{\mathrm{d}}$ & $0.77^{\mathrm{b}}$ & 0.091 \\
\hline 450 & & $4.33^{\mathrm{a}}$ & $1.57^{\mathrm{a}}$ & 0.096 \\
\hline \multicolumn{5}{|l|}{ Enzyme } \\
\hline No & & $3.46^{\mathrm{a}}$ & $0.93^{\mathrm{b}}$ & 0.095 \\
\hline Yes & & $3.44^{\mathrm{b}}$ & $1.13^{\mathrm{a}}$ & 0.092 \\
\hline \multicolumn{5}{|l|}{ Probabilities } \\
\hline Copra & & 0.001 & 0.001 & 0.560 \\
\hline Enzyme & & 0.030 & 0.022 & 0.560 \\
\hline Copra*Enzyme & & 0.001 & 0.001 & 0.560 \\
\hline
\end{tabular}

SFA: saturated fatty acids; MUFA: monounsaturated fatty acids; PUFA: polyunsaturated fatty acids; SEM: standard error of mean; a,b,c: values within the column with different superscripts differ significantly $(\mathrm{P}<0.05)$.

\section{Laying Performance}

Egg performance parameters were not affected by the interaction of CM and enzyme probably due to the similarity in nutrient content of the experimental diets and the lower requirements of egg-type birds. These findings are consistent with those reported by earlier researchers (Panigrahi, 1989) reported no adverse effect of feeding 100 and $200 \mathrm{~g} / \mathrm{kg} \mathrm{CM}$ in Star Cross 288 laying hens. Diarra et al. (2014) reported similar HDP on diets containing $200 \mathrm{~g} \mathrm{CM}$ $/ \mathrm{kg}$ with or without enzyme and control commercial diet in Shaver Brown laying hens. Abeysekara and Atapattu, 2016) also observed no effect of $100 \mathrm{~g} \mathrm{CM} / \mathrm{kg}$ with enzyme in corn-soybean meal based diets on egg production in 13 week-old Japanese quails. Contrary to our results however Moorthy and Viswanathan, (2010) reported lower HDP on $200 \mathrm{~g} \mathrm{CM} / \mathrm{kg}$ in SBM compared to FM-based diets in 21 to 52 week-old single comb white leghorn (SCWL) layers. Dairo and Fasuyi (2008) found that fermented CM diets at 86.5 and $173 \mathrm{~g} / \mathrm{kg}$ produced higher HDP compared to control diet in 37 week-old Black Australorp laying hens. The trend of laying performance in the present study could probably be attributed to possible longer digesta retention of CM-based diets resulting in increased nutrient absorption in the GIT. Diarra et al. (2014) attributed improved laying performance and heavier eggs of hens to longer digesta retention and increased absorption of nutrients. In contrast, Diarra et al. (2018) found no improvement in egg performance of Shaver Brown pullets fed $200 \mathrm{~g} / \mathrm{kg} \mathrm{CM}$ in corn-FM-SBM based diets supplemented with Allzyme SSF (0.3 g/ $/ \mathrm{kg})$ compared to CM diets without enzyme Abeysekara and Atapattu (2016) also found no effect of $0.1 \mathrm{~g} / \mathrm{kg}$ Bio Grain CG enzyme in $200 \mathrm{~g} / \mathrm{kg} \mathrm{CM}$ on egg parameters of Japanese quails. The performance differences among studies could be attributed to several factors including the species, breed, age of birds, CM processing, composition of basal diet, enzyme source and concentration. The higher value of egg shape index on $450 \mathrm{~g} / \mathrm{kg} \mathrm{CM}$ is in agreement with earlier reports. Dairo and Fasuyi (2008) reported higher shape index on $500 \mathrm{~g} / \mathrm{kg}$ fermented CM diet in corn-SBM based diets in 37 weekold Black Australorp laying hens. Krawczyk et al. (2013) found significant effect of feeding diets based on oil seed meals and distillers spent grain on egg shape index in 18 week-old White Leghorn and Rhode Island Red laying hens. The authors also observed lighter yolk colour of eggs on wheat-corn-SBM based diet compared to pigment supplemented control and attributed this to lower xanthophyll and carotenoid content of wheat. Panaitea et al. (2019) observed darker yolk colour score on $75 \mathrm{~g} / \mathrm{kg}$ dried tomato waste in corn-SBM diets compared to control in 96 Tetra SL laying hens and attributed this to the higher carotenoid content of dried tomato waste. The improvement in yolk colour on $450 \mathrm{~g} \mathrm{CM} / \mathrm{kg}$ in this study was not clear but possibly, due to enhancement in carotenoid absorption by the residual fat in CM. Gül et al. (2012) found darker yolk colour in Hisex Brown laying hens fed corn-SBM based diets supplemented with canola oil (20, 40 and $60 \mathrm{~g} / \mathrm{kg}$ ) compared to control.

\section{Fatty Acid Composition of Eggs}

Egg yolks of the groups fed $450 \mathrm{~g} / \mathrm{kg} \mathrm{CM}$ contained higher values of SFA and MUFA. The higher inclusion of $\mathrm{CM}$ and high intake of this diet may explain the pattern of fatty acid in egg yolks because poultry have limited ability to transform dietary fat. Gül et al. (2012) observed higher egg yolk MUFA in Hisex Brown laying hens fed corn- 
SBM based diets with canola oil (20, 40 and $60 \mathrm{~g} / \mathrm{kg}$ ) compared to the control without oil. Recently, Panaitea et al. (2019) found higher egg yolk omega 3 (PUFA) in 96 Tetra SL laying hens fed corn-SBM diets containing 50 $\mathrm{g} / \mathrm{kg}$ dried tomato waste with flaxseed. The authors attributed this to the fatty acid composition of flaxseed and dried tomato waste in the diet and the anti-oxidant property of lycopene content of tomato waste. In an earlier report Cherian and Sim, (1991) also found increased omega 3 fatty acid of flax and canola seeds in eggs compared to control. Panaitea et al. (2019) observed significant effect of diet on fatty acid profile of egg yolk of White Leghorn and Rhode Island Red laying hens fed oil seed meals and distillers spent grain in wheat-cornSBM based diets. Contrary to these findings Farias et al. (2019) found no effect of $250 \mathrm{~g} / \mathrm{kg}$ stored CM corn-SBM diets on egg fatty acid composition of Japanese quails. This suggests that CM quality is an important factor affecting its fatty acid composition. The lower SFA and MUFA despite high FI on $300 \mathrm{~g} / \mathrm{kg} \mathrm{CM}$ diet with enzyme was not be clear and needs further investigation.

\section{Conclusions}

Based on the results, it is concluded that $\mathrm{CM}$ can be included up to $450 \mathrm{~g} / \mathrm{kg}$ in corn-animal protein based diets for pullets and laying hens without compromising pullet growth performance, egg production and quality. Inclusion at 300 and $450 \mathrm{~g} / \mathrm{kg} \mathrm{CM}$ increases FCR in laying hens. Enzyme supplementation reduced egg SFA and increased MUFA composition of eggs. Saturated fatty acids of eggs can be altered at higher CM inclusion with enzyme. Copra meal inclusion will have cost advantage and add value to $\mathrm{CM}$, where it is readily available. More research into the source of CM, composition of basal diet, enzyme source and concentration above $30 \% \mathrm{CM}$ is recommended.

\section{Acknowledgements}

The University of the South Pacific is duly acknowledged for funding the research (Grant no. F5136 RI001-act-prog). Authors are grateful to Professor Velmurugu Ravindran of Massey University, New Zealand and Mr. Ami Sharma of Fiji Ministry of Agriculture Chemistry Laboratory for assisting with chemical analysis of the feed ingredients. Mr. Ratish Chandra's farm, Fiji for provided space and facilities for the research.

\section{Conflict of Interest}

The authors declare no conflict of interest

\section{References}

Abeysekara, T.S., Atapattu, N.S.B.M., 2016.Effects of dietary coconut oil meal with or without an enzyme mixture on laying performance and physical parameters of eggs of Japanese Quail (Coturnix coturnix). Tropic.Agric.Res.27(4):414-419.

AOAC., 1990. Association of Official Analytical Chemists. Official Methods of Analysis 19th edition Washington, DC.

AOAC., 1997.Official Method of Analysis of the Association of Official Analytical Chemists. AOAC Inc., Arlington.

AOAC., 2012.Official Method of Analysis Association of Analytical Chemists.19th Edition, Washington DC, page 121-130.
Boateng, L., Ansong, R., Owusu, W.B., Steiner-Asiedu, M., 2016.Coconut oil and palm oil's role in nutrition, health and national development: A review. Ghana Med.J.50(3):189-196.

Bouvarel, I., Nys, Y., 2013. Optimising egg mass and quality traits in modern laying hens through nutrition. In proceedings 19th European symposium on poultry nutrition. Presented at 19th European symposium on poultry nutrition (ESPN), Potsdam, DEU.

Challenzyme, 2016. Challenzyme 1309A.Beijing Challenge Bio-Technology International Trade Company Limited. Beijing Challenzyme Group, Beijing, China. http://www.challenzyme.com

Cherian, G., 2016.Dietary Manipulation of poultry to develop value-added functional foods for humans. Proceedings of the NZ Poultry Industry Conference, October 4-5 Wellington, New Zealand.13:1-9.

Cherian, G., Sim, J.S., 1991.Effect of feeding full fat flax and canola seeds to laying hens on the fatty acid composition of eggs, embryos and newly hatched chicks.Poult.Sci.70:917-922.

Dairo, F.A.S., Fasuyi, A.O., 2008.Evaluation of fermented palm kernel meal and fermented copra meal proteins as substitute for soybean meal protein in laying hens diets. J. Cent. Eur. Agric. 9(1):35-44.

Devi, A., Diarra, S.S.2019.Maximum utilisation of available resources for efficient poultry feeding in the South Pacific: major issues and prospects. World Poult.Sci.J.75:1-15.

Devi, A., Diarra, S.S., 2017.Influence of dietary protein source and utilisation of copra meal in finishing broiler chicken. Indian J. Anim. Nutr.34(2):193-200.

Devi, A., Diarra, S.S., Mael, S., 2019. Challenzyme supplementation of high expeller copra meal in corn-animal protein diets for broilers: growth performance, nutrient digestibility and carcass traits. Indian J. Anim. Nutr.36(2):187197 http://doi10.5958/2231-6744.2019.00031.8

Diarra, S.S., Kant, R., Tanhimana, J., Lela, P., 2015.Utilisation of Giant African snail (Achatina fulica) meal as protein source for laying hens. J. Agric. Rural Dev. Trop.Subtrop.116(1):85-90.

Diarra, S.S., Kolubalona, J.K., Lal, P.P., Stewart, E., Teine, M.A., Havea, T., Siose, I., Samo, J., Tabi, F., 2018.A Comparative Utilisation of High Dietary Copra and Palm Kernel Cakes Based Diets with Exogenous Enzyme by Young Pullets and Layers. Indian J. Anim. Nutr.35(3):339-344.

Diarra, S.S., Simione, M., Olofia, L., 2014. Performance of Laying Hens Fed High Copra Meal-based Diets with or without Exogenous Enzyme Supplementation. Malay. J. Anim. Sci. 17(2):37-42.

Eisen, E.J., Bohren, B.B., McKean, H.E., 1962.The Haugh unit as a measure of egg albumen quality.Poult.Sci.41:1461-1468.

Farias, N.N.P., Freitas, E.R., do Nascimento, G.A.J., Xavier, R.P.S., de Melo Braz, N., Dantas, F.D.T., Figueiredo, C.W.S., Gomes, V.L.M., Watanabe, P.H., 2019.Fresh and stored copra meal in meat quail diets. Trop. Anim. Health Prod.51(1):179-185.

Fisher, C., Boorman, K.N.1986.Nutrient requirement of poultry and nutritional research: British Poultry Science. Symposium 19, Butherworths, London.

Gül, M., Yörük, A.M., Aksu, T., Kaya, A., Kaynar, Ö., 2012. The effect of different levels of canola oil on performance, egg shell quality and fatty acid composition of laying hens.Int.J.Poult.Sci.11(12):769-776.ISSN 1682-8356 ${ }^{\circ}$

Knudsen, K.E.B., 1997.Carbohydrate and lignin contents of plant materials used in animal feeding. Anim. Feed Sci.Tech.67:319-338

Krawczyk, J., Sokolowicz, Z., Swiatkiewicz, S., Sosin-Bzducha, E., 2013.Effect of outdoor access and increased amounts of local feed materials in the diets of hens covered by the genepool protection programme for farm animals in Poland on quality of eggs during peak egg production. Ann. Anim.Sci.13:327-339. 
Mael, S.H., Diarra, S.S., Devi, A., 2019. Enzyme supplementation of commercial feed diluted with copra meal for growing pullets. Egypt.J.Vet.Sci.50(2):123127.http://doi10.21608/ejvs.2019. 13427.1081

Mateos, G.G., Jimenez-Moreno, E., Serrano, M.P., Lazaro, R.P., 2012. Poultry response to high levels of dietary fiber sources varying in physical and chemical characteristics.J.Appl.Poult.Res.21:156-174.

Moorthy, M., Viswanathan, K., 2006.Feeding Value of Extracted Coconut Meal for White Leghorn Layers.Int.J.Poult.Sci.5(11):1040-1045.

Moorthy, M., Viswanathan, K., 2010.Digestibility and feeding value of coconut meal for white leghorn layers. Tamilnadu, J.Vet.Anim.Sci.6(5):196-203.

NRC., 1994.National Research Council: Nutrient Requirements of Poultry.9th edition. National Academy Press, Washington, D.C.

Panaitea, T.D., Nour, V., Vlaicua, P.A., Ropotaa, M., Corbuc, A.R., Saracila, M., 2019.Flaxseed and dried tomato waste used together in laying hens diet. Arch. Anim. Nutr.73 (3):222-238. https://doi.org/10.1080/1745039X.2019.1586500

Panigrahi, S., 1989.Effects on egg production of including high residual lipid copra meal in laying hen diets.Br.Poult.Sci.30(2):305-312

Panigrahi, S., 1992.Effect of different copra meals and amino acid supplementation on broiler chick growth. Br. Poult. Sci. 33: 675-680.
Pottguetter, R., 2015.Nutrition of hens in extended production cycles - as a practical approach. Proceeding of 16th European Symposium on the Quality of Eggs and Egg Products, Nantes.page 14.

Shaver management guide, 2016.Shaver: profit from efficiency. Accessed 25.02.2020 www.joiceandhill.co.uk

SPSS., 2013.Statistical Package for Social Science.Pc Version, SPSS Inc.444 N. Michigan Avenue Chicago, United States of America.

Steel, R.G.D., Torrie, J.H., 1980.Principles and procedures of statistics.A biometrical approach, 2nd Edition, McGraw Hills Book Co., New York, U.S.A.

Sukhija, P.S., Palmquist, D.L.1988.Rapid method for determination of total fatty acid content and composition of feedstuffs and faeces. J. Agric. Food Chem.36:1202-1206.

Sundu, B., Kumar, A., Dingle, J., 2004.The effect of commercial enzymes on chicks fed high copra meal and palm kernel meal diets. Proceedings Seminar Nasional Pemanfaatan sumber Daya hayati berkelanjutan. Tadulako University Press, Indonesia. Page 26-31.

Sundu, B., Kumar, A., Dingle, J., 2006.Palm kernel meal in broiler diets: effect on chicken performance and health. World Poult.Sci.J.62(2):316-325

Sundu, B., Kumar, A., Dingle, J., 2009.Feeding value of copra meal for Broilers. World Poult.Sci.65:481-491.

Sundu, B., Kumar, A., Dingle, J.G., 2005.Growth pattern of broilers fed a physically or enzymatically treated copra meal diet. Aust. Poult. Sci. Symp.17:291-294. 